九州大学学術情報リポジトリ

Kyushu University Institutional Repository

\title{
Characteristics of myasthenia gravis according to onset-age : Japanese nationwide survey
}

Murai, Hiroyuki

Department of Neurology, I izuka Hospital

Yamashita, Natsumi

Department of Biostatistics, Graduate School of Medicine, Kurume University

Watanabe, Makoto

Department of Preventive Cardiology, National Cardiovascular Center | Department of Public Health, Jichi Medical University

Nomura, Yoshiko

Segawa Neurological Clinic for Children

他

ht tp://hdl. hand le. net/2324/25477

出版情報：Journal of the Neurological Sciences. 305 (1/2)，pp.97-102，2011-06-15. Elsevier バージョン:

権利関係: (C) 2012 Elsevier B.V. 


\section{Characteristics of myasthenia gravis according to onset-age:}

\section{Japanese nationwide survey}

Hiroyuki Murai $^{\mathrm{a}, \mathrm{b}, *}$, Natsumi Yamashita ${ }^{\mathrm{c}}$, Makoto Watanabe ${ }^{\mathrm{d}, \mathrm{h}}$, Yoshiko Nomura $^{\mathrm{e}}$, Masakatsu Motomura ${ }^{\mathrm{f}}$, Hiroaki Yoshikawa ${ }^{\mathrm{g}}$, Yosikazu Nakamura ${ }^{\mathrm{h}}$, Naoki Kawaguchi, Hiroshi Onodera $^{\mathrm{j}}$, Shigeru Araga ${ }^{\mathrm{k}}$, Noriko Isobe ${ }^{\mathrm{a}}$, Masaki Nagai ${ }^{1}$, Jun-ichi Kira ${ }^{\mathrm{a}}$

${ }^{a}$ Department of Neurology, Neurological Institute, Graduate School of Medical Sciences, Kyushu University, Fukuoka, Japan

${ }^{\mathrm{b}}$ Department of Neurology, Iizuka Hospital, Iizuka, Japan

${ }^{c}$ Department of Biostatistics, Graduate School of Medicine, Kurume University, Kurume, Japan

${ }^{\mathrm{d}}$ Department of Preventive Cardiology, National Cardiovascular Center, Suita, Japan

${ }^{\text {e}}$ Segawa Neurological Clinic for Children, Tokyo, Japan

${ }^{\mathrm{f}}$ First Department of Internal Medicine, Graduate School of Biomedical Sciences, Nagasaki

University, Nagasaki, Japan

${ }^{\mathrm{g}}$ Health Service Center, Kanazawa University, Kanazawa, Japan

${ }^{\mathrm{h}}$ Department of Public Health, Jichi Medical University, Shimotsuke, Japan

${ }^{\mathrm{i}}$ Department of Neurology, Graduate School of Medicine, Chiba University, Chiba, Japan 
${ }^{\mathrm{j}}$ Department of Neurology, National Nishitaga Hospital, Sendai, Japan

${ }^{\mathrm{k}}$ Fujii Memorial Hospital, Kurayoshi, Japan

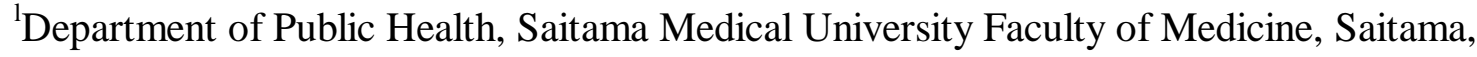
Japan

*Corresponding author. Department of Neurology, Iizuka Hospital, 3-83 Yoshio-machi, Iizuka 820-8505, Japan.

E-mail: hmurai@neuro.med.kyushu-u.ac.jp

Key words: Myasthenia gravis; Epidemiology; Nationwide survey; Onset-age; Infantileonset; Thymoma; Generalized additive model (GAM) 


\section{ABSTRACT}

Objective: To clarify the prevalence and clinical characteristics of myasthenia gravis (MG) in Japan.

Methods: We performed a nationwide epidemiological survey of MG in Japan. The clinical features were compared among five groups of patients, divided according to onset age. A generalized additive model (GAM) was used to assess the linearity of these relationships. Results: A total of 8,542 patients were reported, and detailed data were analyzed for 3,141 patients. The estimated number of MG patients in Japan was 15,100, giving a prevalence of 11.8 per 100,000 . Elderly-onset MG ( $\geq 65$ years) accounted for $7.3 \%$ in 1987 (adjusted for population in 2005), but this had increased to $16.8 \%$ in 2006. Infantile-onset MG (0-4 years) accounted for $10.1 \%$ in 1987 , and was still as high as $7.0 \%$ in 2006 . The rate of ocular MG was highest (80.6\%) in infantile-onset and lowest (26.4\%) in early-onset disease, but the rate rose again in the late-onset group. GAM analysis of the ocular form showed a U-shaped curve, with a dip in the 20s. Anti-acetylcholine receptor antibodies were positive in only $50 \%$ of infantile-onset, but nearly $90 \%$ of elderly-onset patients. GAM analyses assessing the concurrence of thymoma and hyperplasia both showed reversed U-shapes, with peaks in the $50 \mathrm{~s}$ and $20 \mathrm{~s}-40 \mathrm{~s}$, respectively.

Conclusions: Persistent high incidence of infantile-onset disease and clinical heterogeneity according to onset age are characteristic features of MG in Japan. 


\section{Introduction}

Myasthenia gravis (MG) is an autoimmune disease that targets neuromuscular junctions[1]. The disease is characterized by ocular symptoms, bulbar signs, weakness of the extremities, and respiratory failure. The clinical aspects of MG have changed drastically over the past few decades, reflected in decreased mortality [2], along with the discovery of antibodies against muscle-specific tyrosine kinase (MuSK) [3], and the use of immunosuppressants and intravenous immunoglobulin therapy (IVIG).

Several reports have indicated the existence of racial variations in MG, e.g., between patients of Caucasian and African origins. African patients present with a lower positivity for anti-acetylcholine receptor (AChR) antibodies, a higher positivity for anti-MuSK antibodies, and a more severe form of disease, compared with Caucasians [4]. A high frequency of juvenile-onset MG has been described in Asian countries [5-8]; in China, 39-50\% of all MG patients were reportedly children $(<15$ years) $[6,7]$. A high frequency of the ocular from is also a characteristic feature of MG in Asia, ranging from 47-73\% [5-7].

An increasing incidence of late-onset or elderly-onset MG has been reported recently [9-12]. This is a worldwide phenomenon, and is not confined to regional areas of Japan. The increase in late-onset MG has not yet been evaluated at a national level. However, this trend is important, because elderly patients suffer from various complications such as hypertension, diabetes mellitus, hyperlipidemia, stroke, cancer and osteoporosis, and the choice of appropriate therapeutic options can be challenging.

An increased prevalence of autoimmune diseases has been reported in Western developed countries [13-15]. This increase has been considered to be a result of environmental changes and improved medical care. It is therefore important to assess the changes in prevalence and 
clinical features of MG in Asian countries, which have undergone rapid environmental alterations. Japan is the only country in Asia where repeated nationwide epidemiological surveys have been carried out. The previous surveys were performed in 1973 [16, 17] and in 1987 [18]. Because it had been approximately 20 years since the last nationwide survey, we performed a third survey to determine the current epidemiological and clinical traits of MG in Japan.

\section{Methods}

\subsection{Survey procedures}

A nationwide MG survey was conducted by the Research Committees of Neuroimmunological Diseases and of Epidemiology of Intractable Diseases, sponsored by the Ministry of Health, Labour and Welfare, Japan. The study was approved by the Kyushu University Ethics Committee. The survey was carried out in two steps. First, a preliminary survey was undertaken to determine the prevalence and the approximate number of patients with MG in Japan, and a second survey was then conducted using a questionnaire to gather clinical information about each patient. The hospitals included in the study were randomly selected from the directory of all registered hospitals throughout Japan. Selection was made according to a stratification based on the number of beds in each hospital, whereby a higher number of beds led to a higher probability of being selected [19]. The sampling rates were approximately 5\%, 10\%, 20\%, 40\%, 80\%, and 100\% for general hospitals with 20-99 beds, 100-199 beds, 200-299 beds, 300-399 beds, 400-499 beds, and 500 or more beds, respectively. All university hospitals, as well as clinics in which a substantial number of MG patients were seen, were also surveyed. 
The first questionnaire asked for information on the number of MG patients who visited the hospitals between January 1 and December 31, 2005. This was sent to 5,426 departments, including 1,112 neurology/internal medicine, 980 surgery, 841 pediatrics, 808 ophthalmology, 776 otorhinolaryngology, 735 neurosurgery, and 174 cardiac surgery departments.

A second questionnaire was forwarded to the institutions that reported patients in the first survey. This second questionnaire collected detailed clinical information about each patient, including age at onset, sex, birthplace, present address, date of birth, family history, onset symptoms, symptoms at diagnosis, MG Foundation of America (MGFA) classification [20], MG-activities of daily living (ADL) score [21], edrophonium test, repetitive stimulation on electromyography (EMG), anti- AChR antibodies, anti-MuSK antibodies, pathology of thymus and thymoma, complications, selected treatments, and outcome, including MGFA postintervention status [20] and occurrence of crisis (supplemental table). Patients who had identical information for sex, date of birth, and residing prefecture were considered to be duplicates, and one was excluded from the study.

\subsection{Diagnostic criteria}

The diagnostic criteria for MG were attached to the questionnaire. MG was diagnosed when (1) one or more of the following subjective findings was present: ptosis, double vision, limb weakness, swallowing difficulty, speech difficulty, breathing difficulty; (2) one or more of the following objective findings were present: ptosis, ophthalmoplegia, facial weakness, neck weakness, limb and/or truncal weakness, dysphagia, dysarthria, dyspnea; (3) diurnal fluctuation and/or easy fatigability were present; (4) at least one of the following tests was positive: edrophonium test, decremental response on repetitive nerve stimulation, or anti 
AChR antibody; and (5) other conditions were excluded. The excluded conditions were: Lambert-Eaton myasthenic syndrome, muscular dystrophy, polymyositis, periodic paralysis, hyperthyroidism, mitochondrial myopathy, progressive external ophthalmoplegia, GuillainBarré syndrome, polyneuritis, oculomotor palsy, Tolosa-Hunt syndrome, brainstem tumor, brainstem infarction, brainstem encephalitis, herpes and other viral encephalitis, meningitis of the skull base, temporal arteritis, Wernicke's encephalitis, Leigh encephalitis, diabetic ophthalmoplegia, vasculitis, neuro Behcet disease, sarcoidosis, multiple sclerosis, acute disseminated encephalomyelitis, Fisher's syndrome, congenital myasthenic syndrome, congenital myopathy, myotonia, blepharospasm, and apraxia of eye-opening.

\subsection{Onset-age}

Patients with MG were divided into five groups according to the age of onset, and the characteristic clinical features of each group were analyzed. The groups were classified as: infantile-onset (ages 0-4 years), childhood-onset (ages 5-9 years), early-onset (10-49 years), late-onset (50-64 years), and elderly-onset (65 years or older).

\subsection{Statistical analysis}

The estimated number of MG patients in Japan was calculated by adding the figures for the total reported number of patients in each group, divided by the ratio of responding institutions to the number of surveyed institutions for each group. The formulae used to estimate the total number of patients and the $95 \%$ confidence intervals (CIs) have been described in detail elsewhere $[22,23]$. The prevalence rate per 100,000 people was determined based on the population of Japan in 2005. 
The chi-squared test was used to determine the significance of the increase in late- and elderly-onset patients over the two decades. The Kolmogorov-Smirnov two-sample test was used to compare the distributions of onset-age between the 1987 and 2006 studies. Fisher's exact test was used to compare the frequency of patients with familial occurrence in each onset-age group. Univariate analyses were performed using SAS ver. 9.1.3 (SAS Institute, Cary, NC). A generalized additive model (GAM) was fit using R ver. 2.7.0 [24]. GAM was used to assess the linearity (on the logarithm of odds scale) of the relationship between clinical features and age at onset. GAM allows for relaxation of the assumption of linearity by using a flexible smoothing term to fit a smooth curve to the data [25].

\section{Results}

\subsection{Estimated number of patients and prevalence rate}

A total of 3,919 institutions (72.2\%) responded to the initial survey, reporting 8,542 patients with MG. In the second questionnaire, detailed data were collected for 3,141 patients, excluding 15 duplicate cases (36.9\% of the preliminary survey). There were no significant regional differences in response rates. The estimated number of patients with MG was 15,100 (95\% CI: 13,900-16,300), and the estimated prevalence rate was 11.8 per 100,000 (95\% CI: 10.9-12.7). The number of MG patients more than doubled, compared with the results of the nationwide survey in 1987 (Table 1). The male/female ratio was 1/1.7 (63.0\% female), indicating a slight decrease in the proportion of females over the 30 -yearperiod $(67.7 \%$ in 1973 , Table 1$)$.

\subsection{Onset-age distribution}


Fig. 1A shows the onset-age distribution of MG ascertained by the surveys in 1987 and 2006, the former adjusted to the population in 2005 to avoid the influence of the increasing proportion of older people. Infantile-onset MG (onset-age of 0-4 years) accounted for $10.1 \%$ of MG in 1987 and $7.0 \%$ in 2006, which was a strikingly high percentage compared with other onset-ages. This distinctive feature has remained constant over the last 30 years [16-18].

Late- and elderly-onset MG (onset-age of 50 years or older) accounted for $28.8 \%$ of cases in 1987, increasing to $41.7 \%$ in 2006 (Fig. 1A, 1B; p < 0.0001). Elderly-onset MG in particular (onset-age of 65 years or more) accounted for only $7.3 \%$ of MG cases in 1987 , but this rose to $16.8 \%$ in 2006, representing a 2.3-fold increase (Fig. 1B). The significant trend towards a reduction in the proportion of early-onset MG and an increase in that of late- and elderly-onset MG can be clearly observed in Fig. 1C ( $\mathrm{p}<0.0001$ by KolmogorovSmirnov two-sample test).

In addition to the infantile peak, female patients showed two peaks in onset-age, at roughly 30 and 55 years, while male patients demonstrated a linear increase between the ages of 10 and 65 (Fig. 1D).

\section{3. $M G-A D L$ score and MGFA classification}

The MG-ADL score assessed at diagnosis was $5.85 \pm 3.87$ (range 0-24), with a median of 5 (Fig. 1E). The MGFA classification is shown in Fig. 1F. MGFA I (ocular type), II (mild generalized), III (moderate generalized), IV (severe generalized) and V (intubated) accounted for $35.7 \%$, 44.3\% (IIa: 27.8\%, IIb: 16.5\%), 15.6\% (IIIa: 9.0\%, IIIb: 6.6\%), $2.5 \%$ (IVa: $1.1 \%$, IVb: $1.4 \%$ ) and 2.0\%, respectively. MGFA I and II together accounted for $80.0 \%$ of all MG cases. 


\subsection{Family history and clinical symptoms}

Only $0.7 \%$ of cases had a family history of MG, while $3.0 \%$ had a family history of autoimmune disease. The frequencies of the various symptoms at onset were: ptosis, $71.9 \%$; diplopia, 47.3\%; weakness of facial muscles, 5.3\%; bulbar symptoms, $14.9 \%$; weakness of extremities, 23.1\%; and dyspnea, 2.3\%. At diagnosis, these frequencies increased to $81.9 \%$, $59.1 \%, 13.9 \%, 27.6 \%, 44.1 \%$ and $4.9 \%$, respectively. Crises were observed in $7.7 \%$ of cases within 1 year, $3.8 \%$ in $1-5$ years, and $2.9 \%$ in 5 years or more after onset. The overall frequency of crisis was $13.3 \%$ throughout the whole period.

\subsection{Laboratory data and electromyographic studies}

Anti-AChR antibodies were positive at diagnosis in $73.9 \%$ of patients with titers of 93.6 $\pm 296 \mathrm{nmol} / \mathrm{l}$ (range $0.18-7,000)$. Anti-MuSK antibodies were positive in only $1.4 \%$, but were not measured in $94.7 \%$ of cases. The edrophonium test was positive in $75.6 \%$ of cases. A decremental response in EMG at low frequency stimulation was observed in $47.3 \%$ of patients.

\subsection{Thymus and thymoma pathology}

Regarding the residual thymus, hyperplasia was seen in $38.4 \%$ of cases, and normal or involuted thymus was diagnosed in $33.4 \%$. Thymoma was present in $32.0 \%$ of all MG cases. Masaoka stagings of thymomas were as follows: I 36.8\%, II 18.4\%, III 11.8\%, IVa 4.1\%, IVb $1.0 \%$ and unknown $27.8 \%$. WHO classification of the thymomas revealed: $\mathrm{A}, 1.6 \%$; AB, 5.9\%; B1, 7.3\%; B2, 9.7\%; B3, 5.2\%; and unknown, 70.3\%. 


\subsection{Clinical characteristics of each onset-age group}

Patients were classified according to onset-age and parameters were compared among the groups (Table 2). The frequency of familial MG was high (2.38\%) in the infantile-onset group, but did not reach statistical significance $(\mathrm{p}=0.0915)$. The incidence of MGFA I (ocular MG) was as high as $80.6 \%$ in the infantile-onset group, falling to $61.5 \%$ in the childhood-onset group, and $26.4 \%$ in the early-onset group. The incidence of MGFA I increased again in the late- and elderly- onset groups (38.1\% in late-onset, $37.3 \%$ in elderly-

onset group). Edrophonium test positivity was similar in all groups. A decremental response in EMG at low frequency stimulation was observed in only $20.3 \%$ of the infantile-onset group, but it increased with increasing onset-age. Anti-AChR antibodies were positive in approximately half the infantile- and childhood-onset cases, and increased with increasing onset-age. Its positivity was as high as $89.2 \%$ in the elderly-onset group. Thymoma was rare (3.6\%) in the infantile-onset group and infrequent (10.0\%) in the childhood-onset group, but exceeded 30\% in the early-onset group and was particularly frequent in the late-onset group (46.6\%). It decreased again in the elderly-onset group. The percentage of hyperplasia in the residual thymus was high in the early-onset group, but low in the other groups. Treatment options in each onset-age group are summarized in Table 2.

These tendencies were clearly visualized by GAM analyses (Fig. 2). Ocular presentation revealed a clear dip in the 20s (Fig. 2A), and anti-AChR positivity gradually increased along with increasing age (Fig. 2B). The positivity of both thymoma and hyperplasia showed reversed-U shapes with different peaks: thymoma in the 50s and hyperplasia between the $20 \mathrm{~s}$ and 40s (Fig. 2C, 2D).

\section{Discussion}


This study was limited by the relatively low response rate to the second questionnaire (36.9\%); this may have been partly due to the intricate nature of the questionnaire. However, clinical information was collected for 3,141 patients (excluding the duplicates), which represents the largest sample size among the series of nationwide surveys of MG in Japan.

The estimated number of MG patients is steadily increasing along with its prevalence, and it had more than doubled at this survey, compared with that suggested by the 1987 data. This increase is probably due to improved diagnostic accuracy and case ascertainment, and the improved efficacy of treatment and care. The increasing longevity of the population also contributes to this tendency [26]. Nonetheless, elderly-onset MG was definitely shown to have increased, even after adjusting the onset-age distribution of 1987 to the population in 2005.

The incidence of late- and elderly-onset MG is reported to be increasing in Western countries $[9,10]$ and in certain prefectures in Japan $[11,12]$. In the present study, the incidence of MG patients with an onset-age of 50 years or more increased by 1.5 -fold over a 19-year period; elderly-onset patients in particular (onset-age of 65 years or more) showed a 2.3-fold rise. This indicates that elderly-onset MG is the major source of the overall increase. The reason for the increase is unclear, but an immunological background distinct from that in younger patients, and environmental alterations, may be involved. The increased occurrence of thymoma in the late-onset group and the high positivity rate for anti-AChR antibodies in the late- and elderly-onset groups may explain this feature.

The prominent peak in infantile-onset MG (ages 0-4 years) was a notable feature. This peak was also seen in the surveys conducted in 1973 [16, 17] and 1987 (Fig 1A), indicating a persistent tendency. In contrast, there was no peak in childhood-onset disease (onset-age 
5-9 years), which was also the case in the previous surveys. Because this infantile peak remained constant throughout the three decades, it is considered to reflect the genetic background, and is not influenced by the westernized environment. Interestingly, a similar tendency has been reported from China where juvenile MG comprises $39-50 \%$ of cases [6, 7]. These observations suggest that a high proportion of infantile-onset MG cases are a characteristic feature of East Asian populations. Human leukocyte antigen (HLA) analyses may provide clues to the immunopathogenesis of infantile- and childhood-onset MG [27-32]. HLA Bw46 and DR9 have been reported to be associated with juvenile-onset MG in China [27-29], while frequencies of DR9 and DRw13 were significantly increased in infantile-onset MG patients in Japan [30, 31]. This distinct HLA association may contribute to the high frequency of infantile- and juvenile-onset MG in East Asia.

We analyzed the relationship between clinical features and onset-age in this study, by comparing characteristics among the onset-age groups, and by using the GAM method. This method allows easy visualization of the linearity of the relationship between clinical feature and onset-age. The variability in clinical characteristics according to onset-age in Japanese MG patients was notable. The features of infantile-onset MG have not been previously characterized in detail, and no study has focused on the relationship between age of onset and frequencies of waning on repetitive EMG, and anti-AChR antibody positivity. In our study, the infantile-onset group showed a high frequency of ocular form (80.6\%), and lower frequencies of waning on repetitive EMG (20.3\%), anti-AChR antibody (50.3\%), and thymic abnormalities (thymoma 3.6\% and hyperplasia 16.1\%), compared with other onsetage groups. In contrast, early-onset MG patients demonstrated a low frequency of ocular form, a high frequency of thymic hyperplasia, and a high female proportion. In other countries, such as India, the United States, Italy and Brazil, the frequency of the ocular form 
is only $14-30 \%$ and the anti-AChR positivity rates range from $74-82 \%$ in childhood-onset MG [33-36]. A high proportion of childhood-onset (<15 years) or early childhood-onset ( $<10$ years) MG has been reported [5-8] in the Chinese population, together with a high frequency of ocular form $(71-73 \%)[6,7]$ and a relatively low frequency of anti-AChR antibody positivity (64\%) [7]. The unique features of infantile-onset MG in Japan, some of which are shared with Chinese childhood-onset MG, suggest the existence of a distinct immunological mechanism.

Concurrent thymoma was uncommon in the infantile- and childhood-onset groups, but occurred in $33.8 \%$ of the early-onset group, and as many as $46.6 \%$ of the late-onset group. This high rate of thymoma-associated MG in the late-onset group was a characteristic feature of the present survey. The increase in thymoma-associated cases may be related to the increase in MG with an onset-age of 50 years or more, but could also be attributed to the relatively low rate of response to the questionnaire.

The results of this nationwide survey determined that MG in the Japanese population is epidemiologically unique, and that it is clinically diverse, according to the age of onset. Each onset-age group shows distinct characteristics, as demonstrated by GAM analyses. Treatment planning should therefore take onset-age into consideration.

\section{Acknowledgments}

The authors thank Dr. Tetsuro Konishi for providing helpful advice. This work was supported in part by grants from the Neuroimmunological Disease Research Committee and the Epidemiology of Intractable Diseases Research Committee from the Ministry of Health, Labour and Welfare, Japan. 


\section{References}

1. Lewis RA, Selwa JF, Lisak RP. Myasthenia gravis: immunological mechanisms and immunotherapy. Ann Neurol 1995;37 Suppl 1:S51-62.

2. Alshekhlee A, Miles JD, Katirji B, Preston DC, Kaminski HJ. Incidence and mortality rates of myasthenia gravis and myasthenic crisis in US hospitals. Neurology 2009;72:1548-1554.

3. Hoch W, McConville J, Helms S, Newsom-Davis J, Melms A, Vincent A. Autoantibodies to the receptor tyrosine kinase MuSK in patients with myasthenia gravis without acetylcholine receptor antibodies. Nat Med 2001;7:365-368.

4. Oh SJ, Morgan MB, Lu L, Hatanaka Y, Hemmi S, Young A et al. Racial differences in myasthenia gravis in Alabama. Muscle Nerve 2009;39:328-332.

5. Chiu HC, Vincent A, Newsom-Davis J, Hsieh KH, Hung T. Myasthenia gravis: population differences in disease expression and acetylcholine receptor antibody titers between Chinese and Caucasians. Neurology 1987;37:1854-1857.

6. Wong V, Hawkins BR, Yu YL. Myasthenia gravis in Hong Kong Chinese. 2. Paediatric disease. Acta Neurol Scand 1992;86:68-72.

7. Zhang X, Yang M, Xu J, Zhang M, Lang B, Wang W et al. Clinical and serological study of myasthenia gravis in HuBei Province, China. J Neurol Neurosurg Psychiatry 2007;78:386-390.

8. Chiang LM, Darras BT, Kang PB. Juvenile myasthenia gravis. Muscle Nerve 2009;39:423-431.

9. Somnier FE. Increasing incidence of late-onset anti-AChR antibody-seropositive myasthenia gravis. Neurology 2005;65:928-930. 
10. Aarli JA. Myasthenia gravis in the elderly: Is it different? Ann N Y Acad Sci 2008;1132:238-243.

11. Matsuda M, Dohi-Iijima N, Nakamura A, Sekijima Y, Morita H, Matsuzawa S et al. Increase in incidence of elderly-onset patients with myasthenia gravis in Nagano Prefecture, Japan. Intern Med 2005;44:572-577.

12. Matsui N, Nakane S, Nakagawa Y, Kondo K, Mitsui T, Matsumoto T et al. Increasing incidence of elderly onset patients with myasthenia gravis in a local area of Japan. J Neurol Neurosurg Psychiatry 2009;80:1168-1171.

13. Jacobson DL, Gange SJ, Rose NR, Graham NM. Epidemiology and estimated population burden of selected autoimmune diseases in the United States. Clin Immunol Immunopathol 1997;84:223-243.

14. Cooper GS, Stroehla BC. The epidemiology of autoimmune diseases. Autoimmun Rev 2003;2:119-125.

15. Jimenez S, Cervera R, Font J, Ingelmo M. The epidemiology of systemic lupus erythematosus. Clin Rev Allergy Immunol 2003;25:3-12.

16. Hirayama M, Sugishita T, Uono K. Epidemiological study of myasthenia gravis ( in Japanese). Annual Report of Myasthenia Gravis Research Committee 1974:50-66.

17. Uono M. Clinical statistics of myasthenia gravis in Japan. Int J Neurol 1980;14:8799.

18. Takamori M, Igata A. Epidemiological study of myasthenia gravis (in Japanese). Annual Report of Neuroimmunological Disease Research Committee 1988:227-245.

19. Miura K, Nakagawa H, Morikawa Y, Sasayama S, Matsumori A, Hasegawa K et al. Epidemiology of idiopathic cardiomyopathy in Japan: results from a nationwide survey. Heart 2002;87:126-130. 
Murai et al 17

20. Jaretzki A, 3rd, Barohn RJ, Ernstoff RM, Kaminski HJ, Keesey JC, Penn AS et al. Myasthenia gravis: recommendations for clinical research standards. Task Force of the Medical Scientific Advisory Board of the Myasthenia Gravis Foundation of America. Neurology 2000;55:16-23.

21. Wolfe GI, Herbelin L, Nations SP, Foster B, Bryan WW, Barohn RJ. Myasthenia gravis activities of daily living profile. Neurology 1999;52:1487-1489.

22. Hashimoto S, Fukutomi K, Nagai M. Response bias in the nationwide epidemiological survey of an intractable disease in Japan. J Epidemiol 1991;1:27-30.

23. Osoegawa M, Kira J, Fukazawa T, Fujihara K, Kikuchi S, Matsui M et al. Temporal changes and geographical differences in multiple sclerosis phenotypes in Japanese: nationwide survey results over 30 years. Mult Scler 2009;15:159-173.

24. Team RDC. R: A language and environment for statistical computing. Vienna, Austria: R Foundation for Statistical Computing, 2008

25. Hastie TJ, Tibshirani RJ. Generalized Additive Models. New York: Chapman \& Hall/CRC, 1990

26. Meriggioli MN, Sanders DB. Autoimmune myasthenia gravis: emerging clinical and biological heterogeneity. Lancet Neurol 2009;8:475-490.

27. Hawkins BR, Chan-Lui WY, Choi EK, Ho AY. Strong association of HLA BW46 with juvenile onset myasthenia gravis in Hong Kong Chinese. J Neurol Neurosurg Psychiatry 1984;47:555-557.

28. Hawkins BR, Yu YL, Wong V, Woo E, Ip MS, Dawkins RL. Possible evidence for a variant of myasthenia gravis based on HLA and acetylcholine receptor antibody in Chinese patients. Q J Med 1989;70:235-241. 
29. Chen WH, Chiu HC, Hseih RP. Association of HLA-Bw46DR9 combination with juvenile myasthenia gravis in Chinese. J Neurol Neurosurg Psychiatry 1993;56:382385.

30. Matsuki K, Juji T, Tokunaga K, Takamizawa M, Maeda H, Soda M et al. HLA antigens in Japanese patients with myasthenia gravis. J Clin Invest 1990;86:392-399.

31. Nomura Y, Ehara M, Matsuki K, Juji T, Segawa M. HLA haplotype in myasthenia gravis (MG) of Japanese children. Correlative studies between MG patients and their normal siblings and parents. Ann N Y Acad Sci 1993;681:581-583.

32. Shinomiya N, Nomura Y, Segawa M. A variant of childhood-onset myasthenia gravis: HLA typing and clinical characteristics in Japan. Clin Immunol 2004;110:154-158.

33. Ashraf VV, Taly AB, Veerendrakumar M, Rao S. Myasthenia gravis in children: a longitudinal study. Acta Neurol Scand 2006;114:119-123.

34. Rodriguez M, Gomez MR, Howard FM, Jr., Taylor WF. Myasthenia gravis in children: long-term follow-up. Ann Neurol 1983;13:504-510.

35. Evoli A, Batocchi AP, Bartoccioni E, Lino MM, Minisci C, Tonali P. Juvenile myasthenia gravis with prepubertal onset. Neuromuscul Disord 1998;8:561-567.

36. Morita MP, Gabbai AA, Oliveira AS, Penn AS. Myasthenia gravis in children: analysis of 18 patients. Arq Neuropsiquiatr 2001;59:681-685. 


\section{Figure legends}

Fig. 1. Outline of the present survey data compared with data from 1987. (A) Numbers of MG patients in each onset-age group. Each bar represents a width of 5 years, e.g., ' 15 ' indicates an onset-age of 15-19 years. Data from 1987 were adjusted to the population in 2005, to avoid the influence of an increasing older generation. A persistent striking peak exists in the infantile-onset (0-4 years) group. (B) Late- and elderly-onset MG increased over the two decades. The increase in patients with an onset-age of 65 or older was particularly striking. (C) Proportions of patients compared with 1987 data. The decrease in early-onset MG and the increase in late-onset MG are shown $(\mathrm{p}<0.0001$ by KolmogorovSmirnov two-sample test). (D) The sexes of patients in each onset-age group are shown. In addition to the infantile peak, female patients showed two peaks in their 20s and 50s, whereas male patients showed a linear increase between their teens and 60s. (E) The numbers of patients with each MG-ADL score are shown. (F) MGFA classifications of patients are demonstrated. MGFA I and II accounted for $80 \%$ of all MG cases.

Fig. 2. Generalized additive model (GAM) analyses of ocular form, anti-AChR antibody positivity rate, and concurrent thymoma and thymic hyperplasia are shown. We assessed the linearity of the relationships between these clinical features and onset-age. The results are presented as prediction probability, with the shaded region representing the pointwise \pm 1.96 SE band. (A) The incidence of ocular form was the highest in the under 10s, rapidly falling to a dip in the 20 s, then increasing again with increasing onset-age. (B) The anti-AChR antibody positivity rate increased steadily along with increasing onset-age. (C) The presence of concurrent thymoma showed a clean reversed U shape, with a peak in the 50s. (D) 
Murai et al 20

Thymic hyperplasia positivity also showed a reversed U shape, with a wide peak between the $20 \mathrm{~s}$ and $40 \mathrm{~s}$. 
Table 1 Comparison of demographic features among three nationwide surveys

\begin{tabular}{|l|c|c|c|}
\hline & 1973 & 1987 & 2006 \\
\hline Estimated number of patients & ND & 6,000 & 15,100 \\
\hline Prevalence rate (per 100,000) & 1.35 & 5.1 & 11.8 \\
\hline Number of cases collected for the survey & 1,430 & 1,538 & 3,143 \\
\hline Male : female & $1: 2.1$ & $1: 1.85$ & $1: 1.7$ \\
\hline Occurrence within families (\%) & 2.0 & 0.8 & 0.7 \\
\hline Ocular form (\%) & ND & 40.1 & 35.7 \\
\hline Concurrence of thymoma (\%) & 10.6 & 21.1 & 32.0 \\
\hline History of crisis (\%) & 16.0 & 14.8 & 13.3 \\
\hline
\end{tabular}

$\mathrm{ND}=$ not determined 
Table 2 Clinical features of the five onset-age groups

\begin{tabular}{|c|c|c|c|c|c|}
\hline Onset-age group & Infantile-onset & Childhood-onset & Early-onset & Late-onset & Elderly-onset \\
\hline Age at onset & $0-4$ & $5-9$ & $10-49$ & $50-64$ & $65-$ \\
\hline Patient number & 214 & 54 & 1517 & 761 & 515 \\
\hline Male : female & $1: 1.6$ & $1: 1.5$ & $1: 2.3$ & $1: 1.3$ & $1: 1.8$ \\
\hline MG occurrence within families (\%) & 2.38 & 0 & 0.54 & 0.53 & 0.40 \\
\hline MGFA type I (\%) & 80.6 & 61.5 & 26.4 & 38.1 & 37.3 \\
\hline Positive edrophonium test (\%) & 83.9 & 86.3 & 71.5 & 77.1 & 81.0 \\
\hline Positive decremental response (\%) & 20.3 & 36.4 & 49.2 & 48.4 & 51.8 \\
\hline Positive anti-AChR antibodies (\%) & 50.3 & 48.1 & 70.6 & 78.9 & 89.2 \\
\hline Concurrence of thymoma (\%) & 3.6 & 10.0 & 33.8 & 46.6 & 28.4 \\
\hline Hyperplasia in residual thymus (\%) & 16.1 & 21.1 & 46.6 & 30.4 & 21.7 \\
\hline
\end{tabular}


Figure 1

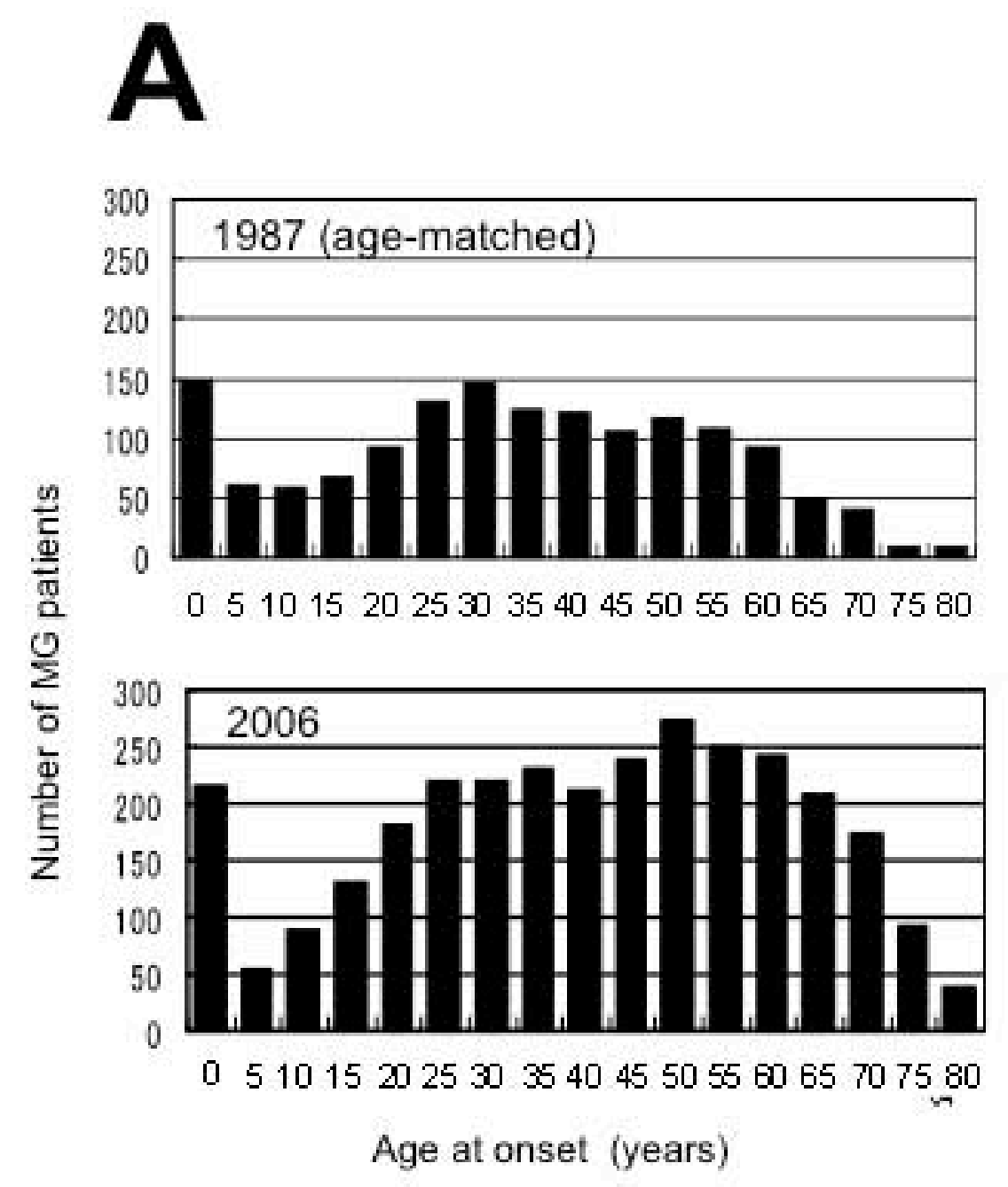

B
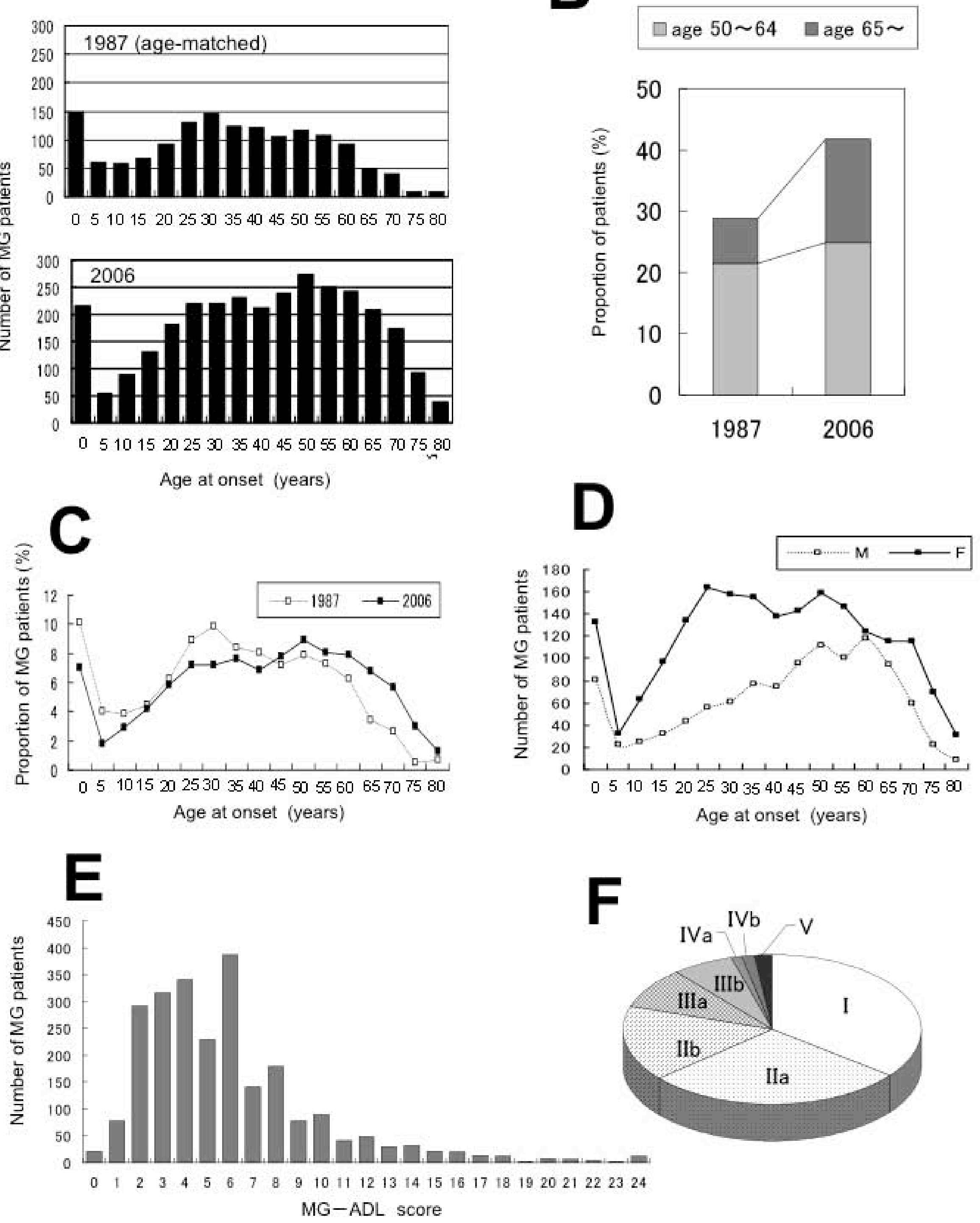
